TOKYO J. MATH.

VoL. 3, No. 1, 1980

\title{
On the Units of Integral Group Rings
}

\author{
Katsusuke SEKIGUCHI \\ Tokyo Metropolitan University \\ (Communicated by K. Ogiue)
}

§ 0. Let $G$ be a finite group and let $Z G$ be its integral group ring. Let $U(\boldsymbol{Z} G)$ denote the unit group of $\boldsymbol{Z} G$ and define $V(\boldsymbol{Z} G)=\{u \in$ $U(Z G) \mid \varepsilon(u)=1\}$ where $\varepsilon: Z G \rightarrow Z$ is the augmentation map. In this paper we will study the following problems:

Problem 1. How many conjugate classes are there in $V(\boldsymbol{Z} G)$ of subgroups of $V(Z G)$ isomorphic to $G$ ?

Problem 2. Is there a torsion free normal subgroup $F$ of $V(Z G)$ such that $V(Z G)=F \cdot G$ ?

Let $S_{n}$ (resp. $A_{n}$ ) denote the symmetric group (resp. alternating group) on $n$ symbols, and let $D_{n}$ denote the dihedral group of order $2 n$.

Hughes and Pearson ([3]) raised Problem 1 with related problems and showed that there is only one conjugate class in $V\left(Z S_{3}\right)$ of subgroups of $V\left(Z S_{3}\right)$ isomorphic to $S_{3}$. Polcino ([6]) showed that there are two conjugate classes in $V\left(Z D_{4}\right)$ of subgroups of $V\left(Z D_{4}\right)$ isomorphic to $D_{4}$. On the other hand, Dennis ([2]) solved affirmatively Problem 2 in the case where $G=S_{3}$. Recently, Miyata ([5]) has solved Problem 2 in the case where $G=D_{n}, n$ odd. He has also solved Problem 1 with an additional hypothesis that the class group of $Z D_{n}$ is of odd order.

Our main results are as follows:

[I] Let $G$ be a finite metabelian group such that the exponent of $G / G^{\prime}$ is $1,2,3,4$ or 6 , where $G^{\prime}$ is the commutator subgroup of $G$. Then there is a torsion free normal subgroup $F$ of $V(Z G)$ such that $V(Z G)=$ $F \cdot G$.

[II] (1) Let $A_{4}$ be the alternating group on 4 symbols. Then there are 4 conjugate classes in $V\left(Z A_{4}\right)$ of subgroups of $V\left(Z A_{4}\right)$ isomorphic to $A_{4}$.

(2) Let $S_{4}$ be the symmetric group on 4 symbols. Then there are 16 conjugate classes in $V\left(Z S_{4}\right)$ of subgroups of $V\left(Z S_{4}\right)$ isomorphic to $S_{4}$.

$\S 1$. Let $G$ be a finite group. For an ideal $J$ of $Z G$, we write 
$U(1+J)=U(Z G) \cap(1+J)$, where $1+J$ is the set of all elements of the form $1+j, j \in J$. For $N \triangleleft G$, denote by $\varepsilon_{G . N}$ the natural map from $Z G$ to $Z(G / N)$ and set $I(G, N)=\operatorname{Ker} \varepsilon_{G . N}$. Note that $\varepsilon_{G . G}$ is the augmentation map of $Z G$ and $V(Z G)=U\left(1+I(G, G)\right.$ ). Write $\varepsilon=\varepsilon_{G . G}$ and $I(G)=I(G, G)$.

We will use the following result.

Proposition 1.1 ([11]). Let $G$ be a finite group and $N \triangleleft G$. Then

$$
N / N^{\prime} \cong I(G, N) / I(G) I(G, N)
$$

under the $\operatorname{map} n N^{\prime} \rightarrow n-1+I(G) I(G, N), n \in N$.

Define the map $U(1+I(G, N)) \rightarrow I(G, N)$ by $1+k \rightarrow k, k \in I(G, N)$. This map induces a group homomorphism

$$
U(1+I(G, N)) / U(1+I(G) I(G, N)) \longrightarrow I(G, N) / I(G) I(G, N) \text {. }
$$

It is easy to see that this is an isomorphism. Therefore we get

COROLlaRY 1.2. Let $G$ be a finite group and $N \triangleleft G$. Then

$$
N / N^{\prime} \cong U(1+I(G, N)) / U(1+I(G) I(G, N)) .
$$

Lemma 1.3 ([4]). Let $G$ be a finite group and let $g \in G$. Then $g-1 \in I(G)^{2}$ if and only if $g \in G^{\prime}$.

Proposition 1.4 ([4]). Suppose that $G$ is a finite metabelian group, then $U\left(1+I(G) I\left(G, G^{\prime}\right)\right.$ ) is a torsion free normal subgroup of $U(Z G)$.

Suppose that $H$ is a finite abelian group. Then, by the theorem of Higman, the only units of finite order in $Z H$ are $\pm h(h \in H)$. It is also known that, if the exponent of $H$ is $1,2,3,4$ or 6 , the only units of $Z H$ are $\pm h(h \in H)$.

TheOREM 1.5. Let $G$ be a finite metabelian group such that the exponent of $G / G^{\prime}$ is $1,2,3,4$ or 6 . Then there is a torsion free normal subgroup $F$ of $V(Z G)$ such that $V(Z G)=F \cdot G$.

Proof. By Corollary 1.2, $U(1+I(G)) / U\left(1+I(G)^{2}\right) \cong G / G^{\prime}$ and

$$
U\left(1+I\left(G, G^{\prime}\right)\right) / U\left(1+I(G) I\left(G, G^{\prime}\right)\right) \cong G^{\prime} .
$$

It is clear that $U\left(1+I\left(G, G^{\prime}\right)\right) \subseteq U\left(1+I(G)^{2}\right)$. Since $G / G^{\prime}$ is an abelian group, $U\left(1+I\left(G / G^{\prime}\right)^{2}\right) \cap\left(G / G^{\prime}\right)=\{1\}$ by Lemma 1.3. Hence, by the assumption on the exponent of $G / G^{\prime}, U\left(1+I\left(G / G^{\prime}\right)^{2}\right)=\{1\}$. Let $\pi: U(Z G) \rightarrow U\left(Z\left(G / G^{\prime}\right)\right)$ be the natural map. Since $\pi\left(U\left(1+I(G)^{2}\right)\right) \leqq U\left(1+I\left(G / G^{\prime}\right)^{2}\right)=\{1\}, U\left(1+I(G)^{2}\right) \subseteq$ 
$\operatorname{Ker} \pi=U\left(1+I\left(G, G^{\prime}\right)\right)$, and therefore $U\left(1+I(G)^{2}\right)=U\left(1+I\left(G, G^{\prime}\right)\right)$. Hence

$$
\begin{aligned}
& \left|V(Z G) / U\left(1+I(G) I\left(G, G^{\prime}\right)\right)\right| \\
& \quad=\left|V(Z G) / U\left(1+I(G)^{2}\right)\right|\left|U\left(1+I\left(G, G^{\prime}\right)\right) / U\left(1+I(G) I\left(G, G^{\prime}\right)\right)\right| \\
& \quad=\left|G / G^{\prime}\right|\left|G^{\prime}\right|=|G| .
\end{aligned}
$$

On the other hand, by Proposition 1.4, $U\left(1+I(G) I\left(G, G^{\prime}\right)\right)$ is a torsion free normal subgroup of $V(Z G)$. Therefore it follows that $U\left(1+I(G) I\left(G, G^{\prime}\right)\right) \cdot G=$ $V(Z G)$. Thus $U\left(1+I(G) I\left(G, G^{\prime}\right)\right)$ is a torsion free normal subgroup of $V(\boldsymbol{Z} G)$, as desired.

REMARK 1. Let $K$ be a finite group. Suppose that there is a torsion free normal subgroup $F$ of $V(Z K)$ such that $V(Z K)=F \cdot K$. Let $H$ be a finite abelian group. Put $G=K \times H$. Then there is a torsion free normal subgroup $\widetilde{F}$ of $V(Z G)$ such that $V(Z G)=\tilde{F} \cdot G$.

Sketch of The Proof. First, we will show that $U(1+I(G, H) I(G))$ is torsion free. Take an element $u$ of finite order in $U(1+I(G, H))$ and write $u=\sum a_{i} h_{i}+\sum_{k_{m} \neq 1} b_{j m} h_{j} k_{m}$, where $h_{i}, h_{j}$ (resp. $k_{m}$ ) range over elements of $H($ resp. $K)$ and $a_{i}, b_{j m} \in Z$. Since $\varepsilon_{G . H}(u)=\sum a_{i}+\sum_{k_{m} \neq 1} b_{j m} k_{m}=1$ in $Z(G / H)$, there exists $a_{i_{0}}$ such that $a_{i_{0}} \neq 0$. Then, by [1, (3.1)], $a_{i_{0}}=1$ and $u=h_{i_{0}} \in H$. For any $h \in H(h \neq 1), h \notin U(1+I(G, H) I(G))$ by Lemma 1.3, hence $U(1+I(G, H) I(G))$ is torsion free. Next, set $\widetilde{F}=U(1+I(G, H) I(G)) \cdot F$. Then $\widetilde{F}$ is torsion free and $[V(Z G): \widetilde{F}]=|G|$. Therefore $\widetilde{F}$ is a torsion free normal subgroup as desired.

REMARK 2. For any finite group $G$ and any integer $n \geqq 3, n \in Z$, consider the natural map $f_{n}: V(Z G) \rightarrow V((Z / n Z) G)$. Then, by $[1,(3.1)]$, $\operatorname{Ker} f_{n}$ is a torsion free normal subgroup of $V(Z G)$ such that $[V(\boldsymbol{Z}(G)$ : $\left.\operatorname{Ker} f_{n}\right]<\infty$. But $\left[V(\boldsymbol{Z} G): \operatorname{Ker} f_{n}\right] \neq|G|$ in general.

REMARK 3. Suppose that $G$ is a finite metabelian group which is a semidirect product of $G^{\prime}$ by a subgroup $H$ of $G$. Since $H$ is an abelian group, by Proposition 1.1, Corollary 1.2 and Lemma 1.3, $U\left(1+I(H)^{2}\right)$ is torsion free and $V(Z H)=H \times U\left(1+I(H)^{2}\right)$. Set

$$
F=U\left(1+I(G) I\left(G, G^{\prime}\right)\right) \cdot U\left(1+I(H)^{2}\right) .
$$

Then $F$ is torsion free and $[V(Z G): F]=|G|$, but it is not a normal subgroup of $V(\boldsymbol{Z} G)$ in general.

$\S 2$. Let $A_{4}$ be the alternating group on 4 symbols $1,2,3$ and 4 . Set $N=\{1,(12)(34),(13)(24),(14)(23)\} \triangleleft A_{4}$ and define $\bar{N}=1+(12)(34)+(13)(24)+$ (14)(23) in $Z A_{4}$. Let $\omega$ be a generator of $A_{4} / N$. 
Hereafter, the unit group of a ring $R$ will be denoted by $U(R)$. Consider the pullback diagram

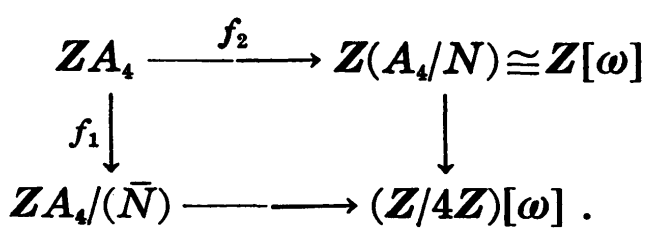

From this diagram we get the Mayer-Vietoris exact sequence (e.g., [7]).

$$
1 \longrightarrow U\left(Z A_{4}\right) \longrightarrow U\left(Z A_{4} /(\bar{N})\right) \times U(Z[\omega]) \longrightarrow U((Z / 4 Z)[\omega]) \longrightarrow 1 \text {. }
$$

The exactness of the last map follows from the fact that $D\left(Z A_{4}\right)=0$ (e.g., [10]). Since $U(Z[\omega])=\left\{ \pm 1, \pm \omega, \pm \omega^{2}\right\}$ we have an exact sequence

$$
1 \longrightarrow U\left(Z A_{4}\right) \longrightarrow U\left(Z A_{4} /(\bar{N})\right) \longrightarrow U((Z / 4 Z)[\omega]) /\langle-1, \omega\rangle \longrightarrow 1 \text {. }
$$

Because $|U((Z / 4 Z)[\omega])|=24,|U((Z / 4 Z)[\omega]) /\langle-1, \omega\rangle|=4$. Define a representation $T$ of $A_{4}$ to $\mathrm{GL}(3, Z)$ by

$$
(12)(34) \longrightarrow\left[\begin{array}{rrr}
1 & 0 & 0 \\
0 & -1 & 0 \\
0 & 0 & -1
\end{array}\right] \text { and }(123) \longrightarrow\left[\begin{array}{lll}
0 & 1 & 0 \\
0 & 0 & 1 \\
1 & 0 & 0
\end{array}\right] \text {. }
$$

Then we can extend $T$ linearly to the map from $Z A_{4}$ to $M_{3}(Z)$ which is denoted by the same symbol $T$. By the map $T$, an arbitrary element $u=a_{1}+a_{2}(12)(34)+a_{3}(13)(24)+a_{4}(14)(23)+b_{1}(123)+b_{2}(243)+b_{3}(142)+b_{4}(134)+$ $c_{1}(132)+c_{2}(234)+c_{3}(124)+c_{4}(143)$ of $Z A_{4}\left(a_{i}, b_{j}, c_{k} \in Z, 1 \leqq i, j, k \leqq 4\right)$ is represented by the matrix

$$
\left[\begin{array}{lll}
a_{1}+a_{2}-a_{3}-a_{4} & b_{1}+b_{2}-b_{3}-b_{4} & c_{1}-c_{2}-c_{3}+c_{4} \\
c_{1}+c_{2}-c_{3}-c_{4} & a_{1}-a_{2}+a_{3}-a_{4} & b_{1}-b_{2}+b_{3}-b_{4} \\
b_{1}-b_{2}-b_{3}+b_{4} & c_{1}-c_{2}+c_{3}-c_{4} & a_{1}-a_{2}-a_{3}+a_{4}
\end{array}\right] .
$$

Putting this matrix to 0 , we get $a_{1}=a_{2}=a_{3}=a_{4}, b_{1}=b_{2}=b_{3}=b_{4}$ and $c_{1}=c_{2}=$ $c_{3}=c_{4}$. Therefore $\operatorname{Ker} T=\bar{N} \cdot Z A_{4}$. Thus $T$ induces an injection from $Z A_{4} /(\bar{N})$ to $M_{3}(Z)$. For $x \in Z A_{4}$, denote by $\bar{x}$ the image of $x$ under the natural map $Z A_{4} \rightarrow Z A_{4} /(\bar{N})$. An arbitrary element $p_{1}+p_{2} \overline{(12)(34)}+$ $p_{3} \overline{(13)(24)}+q_{1}(\overline{123})+q_{2}\left(\overline{12)(34)(123)}+q_{8}(\overline{13)(24})(\overline{123})+r_{1}\left(\overline{132)}+r_{2}(\overline{12)(34)} \overline{(132)}+\right.\right.$ $r_{3} \overline{(13)(24)} \overline{(132)}$ of $Z A_{4} /(\bar{N})\left(p_{i}, q_{j}, r_{k} \in Z, 1 \leqq i, j, k \leqq 3\right)$ is represented by the matrix 
(4)

$$
\left[\begin{array}{lll}
p_{1}+p_{2}-p_{3} & q_{1}+q_{2}-q_{3} & r_{1}+r_{2}-r_{3} \\
r_{1}-r_{2}+r_{3} & p_{1}-p_{2}+p_{3} & q_{1}-q_{2}+q_{3} \\
q_{1}-q_{2}-q_{3} & r_{1}-r_{2}-r_{3} & p_{1}-p_{2}-p_{3}
\end{array}\right]
$$

Viewing $p_{i}$ as variables, the diophantine equation

$$
\left\{\begin{array}{l}
p_{1}+p_{2}-p_{3}=x_{1} \\
p_{1}-p_{2}+p_{3}=x_{2} \\
p_{1}-p_{2}-p_{3}=x_{3}
\end{array}\right.
$$

has a solution in $Z$ if and only if $x_{1} \equiv x_{2} \equiv x_{3}(\bmod 2)$. Applying the same way to $q_{j}$ and $r_{k}$, we get

$$
\begin{aligned}
& U\left(Z A_{4} /(\bar{N})\right) \cong\left\{A \in \mathrm{GL}(3, Z) \mid A \equiv E(\bmod 2), \text { or } A \equiv\left[\begin{array}{lll}
0 & 1 & 0 \\
0 & 0 & 1 \\
1 & 0 & 0
\end{array}\right](\bmod 2),\right. \\
& \text { or } \left.A \equiv\left[\begin{array}{lll}
0 & 0 & 1 \\
1 & 0 & 0 \\
0 & 1 & 0
\end{array}\right](\bmod 2)\right\} .
\end{aligned}
$$

By (1), an element $u=a_{1}+a_{2}(12)(34)+a_{3}(13)(24)+a_{4}(14)(23)+b_{1}(123)+$ $b_{2}(243)+b_{3}(142)+b_{4}(134)+c_{1}(132)+c_{2}(234)+c_{3}(124)+c_{4}(143)$ of $Z A_{4}$ is in $U\left(\boldsymbol{Z} A_{4}\right)$ if and only if $f_{1}(u) \in U\left(Z A_{4} /(\bar{N})\right)$ and $f_{2}(u) \in U(Z[\omega])$. Since $U(Z[\omega])=\left\{ \pm 1, \pm \omega, \pm \omega^{2}\right\}$ and $f_{z}(u)=\sum_{i=1}^{4} a_{i}+\left(\sum_{j=1}^{4} b_{j}\right) \omega+\left(\sum_{k=1}^{4} c_{k}\right) \omega^{2}, u$ is in $U\left(Z A_{4}\right)$ if and only if the matrix of (3) is in $G L(3, Z)$ and $\left(\sum_{i=1}^{4} a_{i}\right.$, $\left.\sum_{j=1}^{4} b_{j}, \sum_{k=1}^{4} c_{k}\right)=( \pm 1,0,0),(0, \pm 1,0)$, or $(0,0, \pm 1)$.

By the same way as in $U\left(Z A_{4} /(\bar{N})\right)$, we get

$$
\begin{aligned}
& U\left(\boldsymbol{Z} A_{4}\right) \cong\left\{\left[\begin{array}{lll}
x_{1} & y_{1} & z_{1} \\
z_{2} & x_{2} & y_{2} \\
y_{3} & z_{3} & x_{3}
\end{array}\right] \in \mathrm{GL}(3, Z) \mid \begin{array}{l}
x_{i} \text { odd, } y_{j}, z_{k} \text { even and } \\
\sum_{j=1}^{3} y_{j} \equiv \sum_{k=1}^{3} z_{k} \equiv 0(\bmod 4)
\end{array},\right. \text { or } \\
& y_{j} \text { odd, } x_{i}, z_{k} \text { even and } z_{k} \text { odd, } y_{j}, x_{i} \text { even and } \\
& \left.\sum_{i=1}^{3} x_{i} \equiv \sum_{k=1}^{3} z_{k} \equiv 0(\bmod 4) \quad \text { or } \quad \sum_{j=1}^{8} y_{j} \equiv \sum_{i=1}^{3} x_{i} \equiv 0(\bmod 4) \quad 1 \leqq i, j, k \leqq 3\right\} \text {. }
\end{aligned}
$$

Let $\psi: \mathrm{GL}(3, \boldsymbol{Z}) \rightarrow \mathrm{GL}(3, \boldsymbol{Z} / 2 Z)$ be the natural map. Since

$$
\psi\left(U\left(Z A_{4} /(\bar{N})\right)\right)=\left\{\left[\begin{array}{lll}
1 & 0 & 0 \\
0 & 1 & 0 \\
0 & 0 & 1
\end{array}\right], \quad\left[\begin{array}{lll}
0 & 1 & 0 \\
0 & 0 & 1 \\
1 & 0 & 0
\end{array}\right], \quad\left[\begin{array}{lll}
0 & 0 & 1 \\
1 & 0 & 0 \\
0 & 1 & 0
\end{array}\right]\right\},
$$


we have $N_{G L(8, z / 2 Z)}\left(\psi\left(U\left(Z A_{4} /(\bar{N})\right)\right)\right)=\psi\left(U\left(U\left(Z A_{4} /(\bar{N})\right)\right) \cdot\left(\left[\begin{array}{lll}0 & 0 & 1 \\ 0 & 1 & 0 \\ 1 & 0 & 0\end{array}\right]\right)\right.$. Hence $N_{G L(3, Z)}\left(U\left(Z A_{4} /(\bar{N})\right)\right)=U\left(Z A_{4} /(\bar{N})\right) \cdot\left(\left[\begin{array}{lll}0 & 0 & 1 \\ 0 & 1 & 0 \\ 1 & 0 & 0\end{array}\right]\right)$. Similarly we see that $N_{G L(3, Z)}\left(U\left(Z A_{4}\right)\right)=U\left(Z A_{4} /(\bar{N})\right) \cdot\left\langle\left[\begin{array}{lll}0 & 0 & 1 \\ 0 & 1 & 0 \\ 1 & 0 & 0\end{array}\right]\right)$.

LEMMA 2.1. $N_{G L(3, Z)}\left(A_{4}\right)= \pm A_{4} \cdot\left(\left[\begin{array}{lll}0 & 0 & 1 \\ 0 & 1 & 0 \\ 1 & 0 & 0\end{array}\right]\right)$.

Proof. First we will show that $U\left(\operatorname{cent}\left(Z_{A_{4}} /(\bar{N})\right)\right)=\{ \pm 1\}$ where $\operatorname{cent}\left(Z A_{4} /\right.$ $(\bar{N}))$ is the center of $Z A_{4} /(\bar{N})$. Let $u=p_{1}+p_{2} \overline{(12)(34)}+p_{3}(\overline{13)(24})+q_{1}(\overline{123})+$ $q_{2}\left(\overline{12)(34)(123)}+q_{3} \overline{(13)(24}\right)(\overline{123})+r_{1}(\overline{132})+r_{2} \overline{(12)(34)}(\overline{132})+r_{3}(\overline{13)(24)}(\overline{132})$ be an element of $U\left(\operatorname{cent}\left(\boldsymbol{Z}_{4} /(\bar{N})\right)\right)$. Since $T(u) \in T\left(\operatorname{cent}\left(\boldsymbol{Q} \otimes_{z} Z A_{4} /(\bar{N})\right)\right)=$ cent $M_{3}(Q), T(u)$ is a diagonal matrix. Hence, in (4), $q_{1}=q_{2}=q_{3}=r_{1}=r_{2}=$ $r_{s}=p_{2}=p_{3}=0$. Therefore $U\left(\operatorname{cent}\left(Z A_{4} /(\bar{N})\right)\right)=\{ \pm 1\}$. Every element of $Z A_{4} /(\bar{N})$ can be written as a $Z$-linear combination of elements in $A_{4}$. So, if an element of $\mathrm{GL}(3, Z)$ normalizes $A_{4}$, then it also normalizes $U\left(Z A_{4} /(\bar{N})\right)$. Thus $N_{G L(3, Z)}\left(A_{4}\right) \subseteq N_{G L(3, Z)}\left(U\left(Z A_{4} /(\bar{N})\right)\right)$. Clearly $\left[\begin{array}{lll}0 & 0 & 1 \\ 0 & 1 & 0 \\ 1 & 0 & 0\end{array}\right] \in N_{G L(3, Z)}\left(A_{4}\right)$. Let $X \in N_{G L(3, Z)}\left(A_{4}\right) \cap U\left(Z A_{4} /(\bar{N})\right)$. Then $X \equiv E$, or $\left[\begin{array}{lll}0 & 1 & 0 \\ 0 & 0 & 1 \\ 1 & 0 & 0\end{array}\right]$, or $\left[\begin{array}{lll}0 & 0 & 1 \\ 1 & 0 & 0 \\ 0 & 1 & 0\end{array}\right](\bmod 2)$. Since $T((123)) \equiv\left[\begin{array}{lll}0 & 1 & 0 \\ 0 & 0 & 1 \\ 1 & 0 & 0\end{array}\right](\bmod 2)$ and $T((132)) \equiv\left[\begin{array}{lll}0 & 0 & 1 \\ 1 & 0 & 0 \\ 0 & 1 & 0\end{array}\right](\bmod 2), X$ acts trivially on $A_{4} / N$. On the other hand, the automorphism group of $A_{4}$ is isomorphic to $S_{4}$. Hence there exists $Y \in$ Aut $A_{4}=S_{4}$ such that $X^{-1} Y$ acts trivially on $A_{4}$. Therefore $Y$ acts trivially on $A_{4} / N$, and hence $Y \in A_{4}$. Since $X^{-1} Y \in\left(\operatorname{cent}\left(Z A_{4} /(\bar{N})\right)\right)=\{ \pm 1\}, X^{-1} Y= \pm 1$. Consequently $X= \pm Y$. This completes the proof.

By the result of [8], there are 3 conjugate classes in $G L(3, Z)$ of subgroups of $\mathrm{GL}(3, Z)$ isomorphic to $A_{4}$. The representatives are as follows:

$$
W_{1}=\left\{\left[\begin{array}{lll}
0 & 1 & 0 \\
0 & 0 & 1 \\
1 & 0 & 0
\end{array}\right],\left[\begin{array}{rrr}
-1 & 0 & 0 \\
0 & 1 & 0 \\
0 & 0 & -1
\end{array}\right]\right\}, \quad W_{2}=\left\{\left[\begin{array}{lll}
0 & 1 & 0 \\
0 & 0 & 1 \\
1 & 0 & 0
\end{array}\right],\left[\begin{array}{lll}
0 & -1 & 1 \\
0 & -1 & 0 \\
1 & -1 & 0
\end{array}\right]\right\},
$$




$$
W_{3}=\left\{\left[\begin{array}{lll}
0 & 1 & 0 \\
0 & 0 & 1 \\
1 & 0 & 0
\end{array}\right], \quad\left[\begin{array}{rrr}
-1 & -1 & -1 \\
0 & 0 & 1 \\
0 & 1 & 0
\end{array}\right]\right\} .
$$

Since, for any $X \in \mathrm{GL}(3, Z), X^{-1} W_{i} X \nsubseteq U\left(Z A_{4} /(\bar{N})\right), i=2$, 3, a subgroup $G$ of $V\left(Z A_{4}\right)$ isomorphic to $A_{4}$ is conjugate to $A_{4}$ in $\mathrm{GL}(3, Z)$. Further $G$ and $A_{4}$ are conjugate in $N_{a L(3, Z)}\left(U\left(Z A_{4}\right)\right)=U\left(Z A_{4} /(\bar{N})\right) \cdot\left(\left[\begin{array}{lll}0 & 0 & 1 \\ 0 & 1 & 0 \\ 1 & 0 & 0\end{array}\right]\right)$.

THEOREM 2.2. There are 4 conjugate classes in $V\left(Z A_{4}\right)$ of subgroups of $V\left(Z_{A_{4}}\right)$ isomorphic to $A_{4}$.

Proof. Let $X, Y$ be elements of $U\left(Z A_{4} /(\bar{N})\right) \cdot\left(\left[\begin{array}{lll}0 & 0 & 1 \\ 0 & 1 & 0 \\ 1 & 0 & 0\end{array}\right]\right)$. Then $X A_{4} X^{-1}$ and $Y A_{4} Y^{-1}$ are conjugate in $V\left(Z A_{4}\right)$ if and only if there exists $Z \in V\left(Z A_{4}\right)$ such

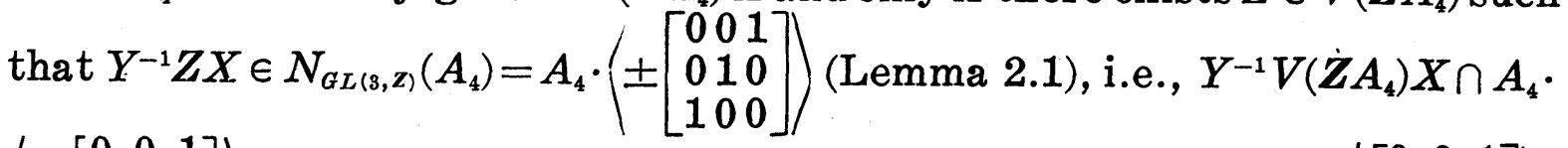
$\left\langle \pm\left[\begin{array}{lll}0 & 0 & 1 \\ 0 & 1 & 0 \\ 1 & 0 & 0\end{array}\right]\right) \neq \phi . \quad$ This condition is equivalent to $X^{-1} Y \in U\left(Z A_{4}\right) \cdot\left(\left[\begin{array}{lll}0 & 0 & 1 \\ 0 & 1 & 0 \\ 1 & 0 & 0\end{array}\right]\right)$. Therefore the number of conjugate classes in $V\left(Z_{4}\right)$ of subgroups

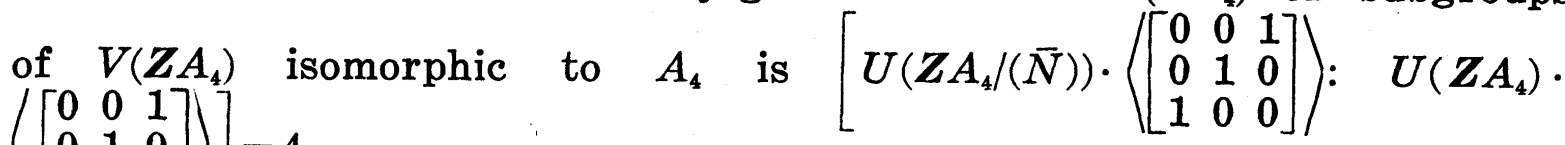
$\left.\left(\left[\begin{array}{lll}0 & 0 & 1 \\ 0 & 1 & 0 \\ 1 & 0 & 0\end{array}\right]\right)\right]=4$.

$\S 3$. In this section, we will consider $S_{4}$, the symmetric group on 4 symbols $1,2,3$ and 4 . We now write $S_{3}=\left\langle\sigma, \tau \mid \sigma^{3}=\tau^{2}=1, \tau \sigma \tau^{-1}=\sigma^{-1}\right\rangle$. Then we have

LEMMA 3.1. $\sigma-\sigma^{-1} \pm \tau-\sigma \tau+\sigma^{2} \tau,-5+2 \sigma+2 \sigma^{2}-4 \sigma \tau+4 \sigma^{2} \tau$ and $1-2 \sigma+$ $2 \sigma^{2}+2 \sigma^{i+1} \tau-2 \sigma^{i+2} \tau, 0 \leqq i \leqq 2$, are units of $Z S_{3}$.

Proof. By direct calculations, we have $\left(\sigma-\sigma^{-1} \pm \tau-\sigma \tau+\sigma^{2} \tau\right)^{-1}=\sigma-$ $\sigma^{-1} \pm \tau-\sigma \tau+\sigma^{2} \tau,\left(-5+2 \sigma+2 \sigma^{2}-4 \sigma \tau+4 \sigma^{2} \tau\right)^{-1}=-5+2 \sigma+2 \sigma^{2}+4 \sigma \tau-4 \sigma^{2} \tau$ and $\left(1-2 \sigma+2 \sigma^{2}+2 \sigma^{i+1}-2 \sigma^{i+2} \tau\right)^{-1}=1+2 \sigma-2 \sigma^{2}-2 \sigma^{i+1} \tau+2 \sigma^{i+2} \tau, 0 \leqq i \leqq 2$.

We note that some of the units in Lemma 3.1 were obtained by Taussky ([9]). Consider the pullback diagram

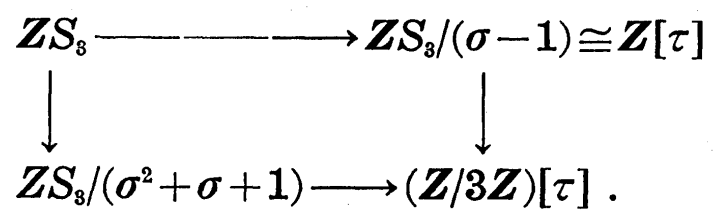


From this diagram we get the exact sequence (5) $1 \longrightarrow U\left(Z S_{8}\right) \longrightarrow U\left(Z S_{3} /\left(\sigma^{2}+\sigma+1\right)\right) \times U(Z[\tau]) \longrightarrow U((Z / 3 Z)[\tau]) \longrightarrow 1$.

The exactness of the last map follows from the fact that $D\left(Z S_{3}\right)=0$.

By [3], there exists a monomorphism $Z S_{3} /\left(\sigma^{2}+\sigma+1\right) \longrightarrow M_{2}(Z)$. An arbitrary element $a_{1}+a_{2} \bar{\sigma}+b_{1} \bar{\tau}+b_{2} \bar{\sigma} \bar{\tau}$ of $Z S_{3} /\left(\sigma^{2}+\sigma+1\right)\left(a_{i}, b_{j} \in Z, 1 \leqq i, j \leqq 2\right)$ is represented by the matrix

$$
\left[\begin{array}{rr}
a_{1}+b_{1} & -a_{2}-b_{1}+b_{2} \\
a_{2}+b_{2} & a_{1}-a_{2}-b_{1}
\end{array}\right]
$$

Therefore $U\left(Z S_{3} /\left(\sigma^{2}+\sigma+1\right)\right) \cong\left\{\left[\begin{array}{ll}a & b \\ c & d\end{array}\right] \in \mathrm{GL}(2, Z) \mid a+c \equiv b+d(\bmod 3)\right\}$. Set $F=\left\{\left[\begin{array}{ll}a & b \\ c & d\end{array}\right] \in \mathrm{GL}(2, Z) \mid a+c \equiv b+d(\bmod 3)\right\}$. By the above discussion we can define a monomorphism $\Psi: U\left(Z S_{3}\right) \rightarrow F \times U(Z[\tau])$.

Consider the commutative diagram

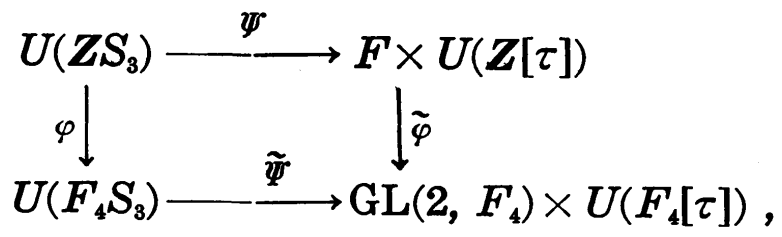

where $F_{4}=Z / 4 Z, \varphi$ and $\widetilde{\varphi}$ are natural maps and $\widetilde{\Psi}$ is induced by $\Psi$. Then $\widetilde{\Psi}$ is an isomorphism.

LEMMA 3.2. $\mid$ Coker $\varphi \mid=4$.

Proof. It is easy to see that the natural map $\mathrm{GL}(2, Z) \rightarrow \mathrm{GL}\left(2, F_{4}\right)$ is surjective. Set $H=\operatorname{Ker}\left\{\mathrm{GL}(2, Z) \rightarrow \mathrm{GL}\left(2, F_{4}\right)\right\}$. Since $F H / H \cong F / F \cap H$, $[F H: F]=[H: F \cap H]$ is a divisor of 4 . Because $\left[\begin{array}{ll}1 & 4 \\ 0 & 1\end{array}\right],\left[\begin{array}{rr}1 & 0 \\ -4 & 1\end{array}\right] \in H \backslash(F \cap H)$ and $\left[\begin{array}{ll}1 & 4 \\ 0 & 1\end{array}\right]^{-1}\left[\begin{array}{rr}1 & 0 \\ -4 & 1\end{array}\right] \notin F \cap H,[H: F \cap H] \geqq 3$. Therefore $[F H: F]=4$ and hence $F H=\mathrm{GL}(2, Z)$. It follows that $F \rightarrow \mathrm{GL}\left(2, F_{4}\right)$ is surjective. Since $U\left(F_{4}[\tau]\right)=$ $\{ \pm 1, \pm \tau, \pm 1+2 \tau, 2 \pm \tau\}, \mid$ Coker $\widetilde{\varphi} \mid=2$. By (5), $\mid$ Coker $\Psi \mid=4$ and $F \times$ $U(Z[\tau])=\operatorname{Im} \Psi \times U(Z[\tau])$. To prove $\mid$ Coker $\varphi \mid=4$, it suffices to show that $\left[\widetilde{\Psi}^{-1}(\operatorname{Im} \widetilde{\varphi}): \operatorname{Im} \varphi\right]=2$. Let $x=\left(\left[\begin{array}{ll}1 & 0 \\ 0 & 1\end{array}\right],-1\right) \in F \times U(Z[\tau])$, then $\widetilde{\Psi}^{-1} \circ \widetilde{\varphi}(x)=$ $-1+2 \sigma+2 \sigma^{2} \in U\left(F_{4} S_{3}\right)$. If we put $u=-5+2 \sigma+2 \sigma^{2}-4 \sigma \tau+4 \sigma^{2} \tau$, then $u \in$ $U\left(Z S_{3}\right)$ by Lemma 3.1 and $\varphi(u)=-1+2 \sigma+2 \sigma^{2}$. Next, we will show that $\widetilde{\Psi}^{-1} \circ \widetilde{\Phi}\left(\left(\left[\begin{array}{ll}1 & 0 \\ 0 & 1\end{array}\right], \tau\right)\right)=2+\sigma+\sigma^{2}-\tau-\sigma \tau-\sigma^{2} \tau \notin \operatorname{Im} \phi$. Suppose conversely that there exists $u \in U\left(Z S_{3}\right)$ such that $\varphi(u)=2+\sigma+\sigma^{2}-\tau-\sigma \tau-\sigma^{2} \tau$. Then we may write $u=2+\sigma+\sigma^{2}-\tau-\sigma \tau-\sigma^{2} \tau+4 f+4 g \tau(f, g \in Z[\sigma])$. Since $\varepsilon_{\left.S_{3}<\sigma\right\rangle}(u) €$ 
$U(Z[\tau])=\{ \pm 1, \pm \tau\}, f$ and $g$ must be written as $f=a_{0}+a_{1} \sigma+\left(-1-a_{0}\right.$ $\left.a_{1}\right) \sigma^{2}, g=b_{0}+b_{1} \sigma+\left(1-b_{0}-b_{1}\right) \sigma^{2}$ for some $a_{i}, b_{j} \in Z$. Therefore $u=2+4 a_{0}+$ $\left(1+4 a_{1}\right) \sigma+\left(-3-4 a_{0}-4 a_{1}\right) \sigma^{2}+\left(-1+4 b_{0}\right) \tau+\left(-1+4 b_{1}\right) \sigma \tau+\left(3-4 b_{0}-4 b_{1}\right) \sigma^{2} \tau$. Hence we have

$$
\Psi(u)=\left(\left[\begin{array}{ll}
1+4\left(2 a_{0}+a_{1}+2 b_{0}+b_{1}\right) & -4\left(1+a_{0}+2 a_{1}+b_{0}-b_{1}\right) \\
4\left(a_{0}+2 a_{1}+b_{0}+2 b_{1}\right) & 1+4\left(1+a_{0}-a_{1}-2 b_{0}-b_{1}\right)
\end{array}\right], \tau\right) .
$$

Since the determinant of this matrix is $1+4\left(1+3 a_{0}\right)+16\left(2 a_{0}+a_{1}+2 b_{0}+b_{1}\right)$ $\left(1+a_{0}-a_{1}-b_{1}\right)+16\left(1+a_{0}+2 a_{1}+b_{0}-b_{1}\right)\left(a_{0}+2 a_{1}+b_{0}+2 b_{1}\right)$, we get $1+3 a_{0}+$ $4\left(2 a_{0}+a_{1}+2 b_{0}+b_{1}\right)\left(1+a_{0}-a_{1}-2 b_{0}-b_{1}\right)+4\left(1+a_{0}+2 a_{1}+b_{0}-b_{1}\right)\left(a_{0}+2 a_{1}+b_{0}+\right.$ $\left.2 b_{1}\right)=0$. But this is equal to $1+3 a_{0}+12\left(a_{0}^{2}+a_{0}+a_{0} a_{1}+a_{1}+b_{0}+b_{1}+a_{1}^{2}-b_{0}^{2}-\right.$ $\left.b_{0} b_{1}-b_{1}^{2}\right)$ which is a contradiction. Therefore $\left[\widetilde{\Psi}^{-1}(\operatorname{Im} \widetilde{\varphi}): \operatorname{Im} \varphi\right]=2$.

Set $N_{1}=\left\{1,1+2 \sigma^{i}\left(\sigma+\sigma^{2}\right) \tau, 1+2\left(\sigma+\sigma^{2}\right), 1+2\left(\sigma+\sigma^{2}\right)+2 \sigma^{i}\left(\sigma+\sigma^{2}\right) \tau, 0 \leqq\right.$ $i \leqq 2\} \leqq U\left(F_{4} S_{8}\right)$ and $N_{2}=\left\langle\sigma-\sigma^{2}+\tau-\sigma \tau+\sigma^{2} \tau\right\rangle$. Then $N_{1}$ (resp. $\left.N_{2}\right)$ is a subgroup of $U\left(F_{4} S_{3}\right)$ of order 8 (resp. 2). A direct calculation shows that $\sigma-\sigma^{2}+\tau-\sigma \tau+\sigma^{2} \tau$ is commutative with each element of $N_{1}$.

Corollary 3.3. $\operatorname{Im} \varphi=\left( \pm N_{1} \times N_{2}\right) \cdot S_{3}$.

Proof. By Lemma 3.1, $\left( \pm N_{1} \times N_{2}\right) \cdot S_{3} \subseteq \operatorname{Im} \varphi$. But $\left[U\left(F_{4} S_{3}\right):\left( \pm N_{1} \times\right.\right.$ $\left.\left.N_{2}\right) \cdot S_{3}\right]=4$. Therefore, by Lemma 3.2, $\operatorname{Im} \phi=\left( \pm N_{1} \times N_{2}\right) \cdot S_{3}$. gram

Let $S_{4} \triangleright N=\{1,(12)(34),(13)(24),(14)(23)\}$. Consider the pullback dia-

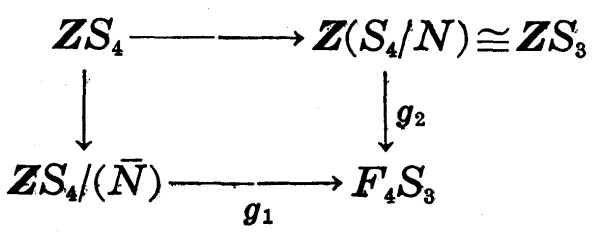

where $F_{4}=\boldsymbol{Z} / 4 \boldsymbol{Z}$. From this diagram we get the exact sequence

$$
U^{*}\left(Z S_{4} /(\bar{N})\right) \times U^{*}\left(Z S_{3}\right) \stackrel{\rho}{\longrightarrow} U\left(F_{4} S_{3}\right) \longrightarrow 1,
$$

where $U^{*}\left(Z S_{4} /(\bar{N})\right)\left(\right.$ resp. $\left.U^{*}\left(Z S_{3}\right)\right)$ denotes the image of $U\left(Z S_{4} /(\bar{N})\right)($ resp. $\left.U\left(Z S_{3}\right)\right)$ in $U\left(F_{4} S_{8}\right)$. The exactness of $\rho$ follows from the fact that $D\left(Z S_{4}\right)=0 \quad$ (e.g., [10]). Since $U^{*}\left(Z S_{4} /(\bar{N})\right) \supseteq U^{*}\left(Z S_{3}\right), \rho\left(U^{*}\left(Z S_{4} /(\bar{N})\right)\right)=$ $U\left(F_{4} S_{3}\right)$. We also have an exact sequence

$$
1 \longrightarrow U\left(Z S_{4}\right) \longrightarrow U\left(Z S_{4} /(\bar{N})\right) \times U\left(Z S_{3}\right) .
$$

Define a representation $T_{1}$ of $Z S_{4}$ to $M_{3}(Z) \oplus M_{3}(Z)$ by 


$$
\begin{aligned}
T_{1}((12)) & =\left(\left[\begin{array}{lll}
1 & 0 & 0 \\
0 & 0 & 1 \\
0 & 1 & 0
\end{array}\right],\left[\begin{array}{rrr}
-1 & 0 & 0 \\
0 & 0 & -1 \\
0 & -1 & 0
\end{array}\right]\right), \\
T_{1}((1234)) & =\left(\left[\begin{array}{rrr}
0 & 0 & -1 \\
0 & -1 & 0 \\
1 & 0 & 0
\end{array}\right],\left[\begin{array}{rrr}
0 & 0 & 1 \\
0 & 1 & 0 \\
-1 & 0 & 0
\end{array}\right]\right) .
\end{aligned}
$$

Then we see that $\operatorname{Ker} T_{1}=\bar{N} \cdot Z S_{4}$. Thus $T_{1}$ induces an injection from $Z S_{4} /(\bar{N})$ to $M_{3}(Z) \oplus M_{8}(Z)$. For $x \in Z S_{4}$, denote by $\bar{x}$ the image of $x$ under the natural map $Z S_{4} \rightarrow Z S_{4} /(\bar{N})$. Then an arbitrary element

$$
\begin{aligned}
& u=a_{1}+a_{2}\left(\overline{12)(34)}+a_{8}\left(\overline{13)(24)}+b_{1}(\overline{123})+b_{2}(\overline{12)(34})(\overline{123})+b_{3}(\overline{13)(24})(\overline{123})\right.\right. \\
& +c_{1}(\overline{132})+c_{2}\left(\overline{12)(34)}(\overline{132})+c_{3}(\overline{13)(24)} \overline{(132})+d_{1}(\overline{12})+d_{2}(\overline{12)(34)}(\overline{12})\right. \\
& +d_{3}\left(\overline{13)(24)}(\overline{12})+e_{1}(\overline{13})+e_{2} \overline{(12)(34)}(\overline{13})+e_{3}\left(\overline{13)(24)}(\overline{13})+f_{1}(\overline{23})\right.\right. \\
& \left.+f_{2} \overline{(12)(34)} \overline{(23}\right)+f_{8}(\overline{13)(24)}(\overline{23})
\end{aligned}
$$

of $Z S_{4} /(\bar{N})\left(a_{i}, b_{j}, c_{k}, d_{l}, e_{m}, f_{n} \in Z, 1 \leqq i, j, k, l, m, n \leqq 3\right)$ is represented by the matrices

where

$$
\left(\left[\begin{array}{lll}
a_{11} & a_{12} & a_{13} \\
a_{21} & a_{22} & a_{23} \\
a_{31} & a_{32} & a_{33}
\end{array}\right],\left[\begin{array}{lll}
b_{11} & b_{12} & b_{13} \\
b_{21} & b_{22} & b_{23} \\
b_{31} & b_{32} & b_{33}
\end{array}\right]\right)
$$

$$
\begin{aligned}
& a_{11}=a_{1}+a_{2}-a_{3}+d_{1}+d_{2}-d_{3}, a_{12}=b_{1}+b_{2}-b_{3}+f_{1}+f_{2}-f_{3}, \\
& a_{13}=c_{1}+c_{2}-c_{3}+e_{1}+e_{2}-e_{3}, a_{21}=c_{1}-c_{2}+c_{3}+f_{1}-f_{2}+f_{8}, \\
& a_{22}=a_{1}-a_{2}+a_{3}+e_{1}-e_{2}+e_{3}, a_{23}=b_{1}-b_{2}+b_{3}+d_{1}-d_{2}+d_{3}, \\
& a_{31}=b_{1}-b_{2}-b_{3}+e_{1}-e_{2}-e_{3}, a_{32}=c_{1}-c_{2}-c_{3}+d_{1}-d_{2}-d_{3}, \\
& a_{33}=a_{1}-a_{2}-a_{3}+f_{1}-f_{2}-f_{3}, b_{11}=a_{1}+a_{2}-a_{3}-d_{1}-d_{2}+d_{8}, \\
& b_{12}=b_{1}+b_{2}-b_{3}-f_{1}-f_{2}+f_{8}, b_{13}=c_{1}+c_{2}-c_{8}-e_{1}-e_{2}+e_{3}, \\
& b_{21}=c_{1}-c_{2}+c_{3}-f_{1}+f_{2}-f_{3}, b_{22}=a_{1}-a_{2}+a_{3}-e_{1}+e_{2}-e_{3}, \\
& b_{23}=b_{1}-b_{2}+b_{3}-d_{1}+d_{2}-d_{3}, b_{31}=b_{1}-b_{2}-b_{3}-e_{1}+e_{2}+e_{3}, \\
& b_{32}=c_{1}-c_{2}-c_{3}-d_{1}+d_{2}+d_{3} \text { and } b_{33}=a_{1}-a_{2}-a_{3}-f_{1}+f_{2}+f_{8} .
\end{aligned}
$$

Put $\widetilde{E}=\left[\begin{array}{lll}1 & 1 & 1 \\ 1 & 1 & 1 \\ 1 & 1 & 1\end{array}\right]$. Then we have

$$
U\left(Z S_{4} /(\bar{N})\right) \cong\left\{\begin{array}{l|l}
A_{1} \times A_{2} \in \mathrm{GL}(3, Z)^{2} & \begin{array}{l}
A_{1} \equiv A_{2} \equiv E(\bmod 2) \\
\text { and } \\
A_{1}-A_{2} \equiv 0 \text { or } 2 \widetilde{E}(\bmod 4),
\end{array}
\end{array}\right.
$$


or $\begin{gathered}A_{1} \equiv A_{2} \equiv \\ \text { and }\end{gathered}\left[\begin{array}{lll}0 & 1 & 0 \\ 0 & 0 & 1 \\ 1 & 0 & 0\end{array}\right](\bmod 2)$,

$A_{1}-A_{2} \equiv 0$ or $2 \widetilde{E}(\bmod 4)$

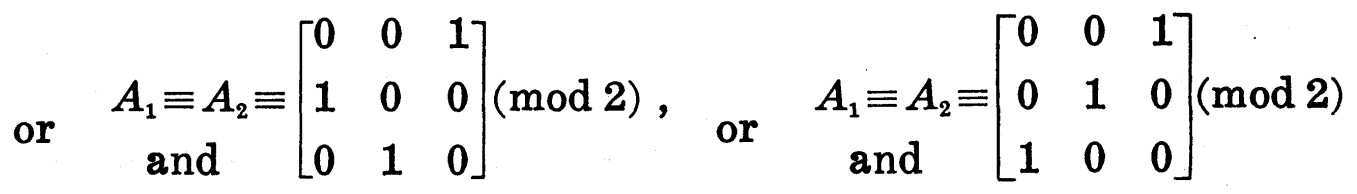

$A_{1}-A_{2} \equiv 0$ or $2 \widetilde{E}(\bmod 4) \quad A_{1}+A_{2} \equiv 0$ or $2 \widetilde{E}(\bmod 4)$

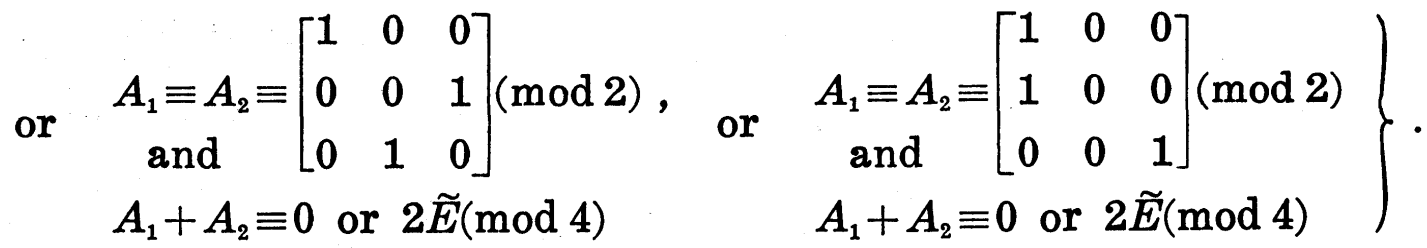

Set $\quad U_{0}\left(Z S_{4} /(\bar{N})\right)=g_{1}^{-1}\left(g_{2}\left(U\left(Z S_{3}\right)\right)\right) \cap U\left(Z S_{4} /(\bar{N})\right)$ and $V_{0}\left(Z S_{4} /(\bar{N})\right)=$ $g_{1}^{-1}\left(g_{2}\left(V\left(Z S_{3}\right)\right)\right) \cap U\left(Z S_{4} /(\bar{N})\right)$. Then $\left[U\left(Z S_{4} /(\bar{N})\right): V_{0}\left(Z S_{4} /(\bar{N})\right)\right]=8$ by Lemma 3.2 and $V\left(Z S_{4}\right)=\left\{\left(v_{1}, v_{2}\right) \in V_{0}\left(Z S_{4} /(\bar{N})\right) \times V\left(Z S_{3}\right) \mid g_{1}\left(v_{1}\right)=g_{2}\left(v_{2}\right)\right\}$.

Since every element of $U\left(Z S_{4} /(\bar{N})\right)$ is a $Z$-linear combination of elements of $V_{0}\left(Z S_{4} /(\bar{N})\right), N_{G L(s, Z)^{2}}\left(V_{0}\left(Z S_{4} /(\bar{N})\right)\right) \subseteq N_{G L(s, Z)^{2}}\left(U\left(Z S_{4} /(\bar{N})\right)\right)$ and we get $N_{G L(3, Z)^{2}}\left(U\left(Z S_{4} /(\bar{N})\right)\right)=U\left(Z S_{4} /(\bar{N})\right) \cdot\left\langle\left(E,\left[\begin{array}{rrr}1 & 2 & 2 \\ -2 & -3 & -2 \\ 2 & 2 & 1\end{array}\right]\right)\right\rangle$. We will show that $N_{G L(3, Z)^{2}}\left(V_{0}\left(Z S_{4} /(\bar{N})\right)\right)=N_{G L(3, Z) 2}\left(U\left(Z S_{4} /(\bar{N})\right)\right)$. Since $g_{2}\left(V\left(Z S_{8}\right)\right) \triangleleft U\left(F_{4} S_{3}\right)$, $U\left(Z S_{4} /(\bar{N})\right) \subseteq N_{G L(8, Z)^{2}}\left(V_{0}\left(Z S_{4} /(\bar{N})\right)\right)$. To show that $\left(\left(E,\left[\begin{array}{rrr}1 & 2 & 2 \\ -2 & -3 & -2 \\ 2 & 2 & 1\end{array}\right]\right)\right) \epsilon$ $N_{G L(3, Z) 2}\left(V_{0}\left(Z S_{4} /(\bar{N})\right)\right)$, let $u$ be an element of $U\left(Z S_{4} /(N)\right)$ which is represented as in (6). By a direct calculation, we have

$$
\left(E,\left[\begin{array}{rrr}
1 & 2 & 2 \\
-2 & -3 & -2 \\
2 & 2 & 1
\end{array}\right]\right) T_{1}(u)\left(E,\left[\begin{array}{rrr}
1 & 2 & 2 \\
-2 & -3 & -2 \\
2 & 2 & 1
\end{array}\right]^{-1}\right)=T_{1}(u)+\left(0,\left(\alpha_{i j}\right)\right)
$$

for some $\left(\alpha_{i j}\right) \in M_{3}(Z)$ such that

$$
\begin{aligned}
& \alpha_{11}+\alpha_{22}+\alpha_{33} \equiv \alpha_{12}+\alpha_{23}+\alpha_{31} \equiv \alpha_{18}+\alpha_{21}+\alpha_{82} \\
& \quad \equiv \alpha_{11}+\alpha_{28}+\alpha_{82} \equiv \alpha_{12}+\alpha_{21}+\alpha_{33} \equiv \alpha_{13}+\alpha_{22}+\alpha_{31} \equiv 0(\bmod 8)
\end{aligned}
$$

Let $v=p_{11}+p_{12} \overline{(2)(34)}+p_{18}\left(\overline{13)(24)}+p_{21}(\overline{123})+p_{22}\left(\overline{12)(34)}(\overline{123})+p_{23}(\overline{13)(24})(\overline{123})+\right.\right.$ $p_{31}(\overline{132})+p_{32}(\overline{12)(34})(\overline{132})+p_{33}(\overline{13)(24})(\overline{132})+p_{41}(\overline{12})+p_{42}(\overline{12)(34})(\overline{12})+$ $p_{43}(\overline{13)(24})(\overline{12})+p_{51}(\overline{13})+p_{52}(\overline{12)(34})(\overline{13})+p_{53}(\overline{13)(24})(\overline{13})+p_{61}(\overline{23})+$ 
$p_{62} \overline{(12)(34)}(\overline{23})+p_{63}\left(\overline{13)(24)}(\overline{23})\left(p_{i j} \in Z, 1 \leqq i \leqq 6,1 \leqq j \leqq 3\right)\right.$ be the element of $U\left(Z S_{4} /(\bar{N})\right)$ such that $T_{1}(v)=\left(0,\left(\alpha_{i j}\right)\right)$.

Comparing the diagonal entries, we have

$$
\begin{aligned}
& p_{11}+p_{12}-p_{13}+p_{41}+p_{42}-p_{43}=0 \\
& p_{11}-p_{12}+p_{13}+p_{51}-p_{52}+p_{53}=0 \\
& p_{11}-p_{12}-p_{13}+p_{61}-p_{62}-p_{63}=0 \\
& 3 p_{11}-p_{12}-p_{13}-p_{41}-p_{42}+p_{43}-p_{61}+p_{52}-p_{63}-p_{61}+p_{62}+p_{63} \equiv 0(\bmod 8) .
\end{aligned}
$$

From this equation it is easy to see that $p_{11}+p_{12}+p_{18} \equiv 0(\bmod 4)$. Similarly we get $p_{i 1}+p_{i 2}+p_{i 3} \equiv 0(\bmod 4)(2 \leqq i \leqq 6)$, hence $g_{1}\left(T_{1}^{-1}\left(0,\left(\alpha_{i j}\right)\right)\right)=0 \in U\left(F_{4} S_{3}\right)$. Therefore $\left(\left(E,\left[\begin{array}{rrr}1 & 2 & 2 \\ -2 & -3 & -2 \\ 2 & 2 & 1\end{array}\right]\right)\right) \in N_{G L(3, Z) 2}\left(V_{0}\left(Z S_{4} /(\bar{N})\right)\right)$. Thus we get $N_{G L\left(3, Z^{2}\right)^{2}}\left(V_{o}\left(Z S_{4} /(\bar{N})\right)\right)=U\left(Z S_{4} /(\bar{N})\right) \cdot\left(\left(E,\left[\begin{array}{rrr}1 & 2 & 2 \\ -2 & -3 & -2 \\ 2 & 2 & 1\end{array}\right]\right)\right)$.

By the result of [8], there are 6 conjugate classes in $\operatorname{GL}(3, Z)$ of subgroups of $\mathrm{GL}(3, Z)$ isomorphic to $S_{4}$. The representatives are as follows:

$$
\begin{aligned}
& W_{1}=\left\{\left[\begin{array}{rrr}
0 & 0 & 1 \\
0 & 1 & 0 \\
-1 & 0 & 0
\end{array}\right],\left[\begin{array}{rrr}
-1 & 0 & 0 \\
0 & 0 & -1 \\
0 & -1 & 0
\end{array}\right]\right\}, W_{2}=\left\{\left[\begin{array}{rrr}
0 & 0 & -1 \\
0 & -1 & 0 \\
1 & 0 & 0
\end{array}\right],\left[\begin{array}{lll}
1 & 0 & 0 \\
0 & 0 & 1 \\
0 & 1 & 0
\end{array}\right]\right\}, \\
& W_{3}=\left\{\left[\begin{array}{rrr}
0 & -1 & 0 \\
1 & 1 & 1 \\
-1 & 0 & 0
\end{array}\right],\left[\begin{array}{rrr}
-1 & -1 & 0 \\
0 & 1 & 0 \\
0 & 0 & -1
\end{array}\right]\right\}, W_{4}=\left\{\left[\begin{array}{rrr}
0 & 1 & 0 \\
-1 & -1 & -1 \\
1 & 0 & 0
\end{array}\right],\left[\begin{array}{rrr}
1 & 1 & 0 \\
0 & -1 & 0 \\
0 & 0 & 1
\end{array}\right]\right\}, \\
& W_{5}=\left\{\left[\begin{array}{rrr}
1 & 1 & 0 \\
-2 & -1 & -1 \\
0 & 0 & 1
\end{array}\right],\left[\begin{array}{rrr}
-1 & -1 & -1 \\
0 & 0 & 1 \\
0 & 1 & 0
\end{array}\right]\right\}, W_{6}=\left\{\left[\begin{array}{rrr}
-1 & -1 & 0 \\
2 & 1 & 1 \\
0 & 0 & -1
\end{array}\right],\left[\begin{array}{rrr}
1 & 1 & 1 \\
0 & 0 & -1 \\
0 & -1 & 0
\end{array}\right]\right\} .
\end{aligned}
$$

We see that there are two conjugate classes in $\mathrm{GL}(3, Z)^{2}$ of subgroups of $\mathrm{GL}(3, Z)^{2}$ isomorphic to $S_{4}$ which are not trivially intersected with $U\left(Z S_{4} /(\bar{N})\right)$. The representatives are as follows:

$$
\begin{aligned}
& L_{1}=\left\{\left(\left[\begin{array}{rrr}
0 & 0 & -1 \\
0 & -1 & 0 \\
1 & 0 & 0
\end{array}\right],\left[\begin{array}{rrr}
0 & 0 & 1 \\
0 & 1 & 0 \\
-1 & 0 & 0
\end{array}\right]\right),\left(\left[\begin{array}{lll}
1 & 0 & 0 \\
0 & 0 & 1 \\
0 & 1 & 0
\end{array}\right],\left[\begin{array}{rrr}
-1 & 0 & 0 \\
0 & 0 & -1 \\
0 & -1 & 0
\end{array}\right]\right)\right\}, \\
& L_{2}=\left\{\left(\left[\begin{array}{rrr}
0 & 0 & 1 \\
0 & 1 & 0 \\
-1 & 0 & 0
\end{array}\right],\left[\begin{array}{rrr}
0 & 0 & -1 \\
0 & -1 & 0 \\
1 & 0 & 0
\end{array}\right]\right),\left(\left[\begin{array}{rrr}
-1 & 0 & 0 \\
0 & 0 & -1 \\
0 & -1 & 0
\end{array}\right],\left[\begin{array}{lll}
1 & 0 & 0 \\
0 & 0 & 1 \\
0 & 1 & 0
\end{array}\right]\right)\right\}
\end{aligned}
$$


Hence the conjugate class containing $L_{1}$ is the only one class which is not trivially intersected with $V_{0}\left(Z S_{4} /(\bar{N})\right)$. Therefore any subgroup $G$ of $V_{o}\left(Z S_{4} /(\bar{N})\right)$ isomorphic to $S_{4}$ is conjugate to $S_{4}$ in $G L(3, Z)^{2}$. Further, $G$ is conjugate to $S_{4}$ in $U\left(Z S_{4} /(\bar{N})\right) \cdot\left(\left(E,\left[\begin{array}{rrr}1 & 2 & 2 \\ -2 & -3 & -2 \\ 2 & 2 & 1\end{array}\right]\right)\right)$.

Lemma 3.4. There are 8 conjugate classes in $V_{0}\left(Z S_{4} /(\bar{N})\right)$ of subgroups of $V_{0}\left(Z S_{4} /(\bar{N})\right)$ isomorphic to $S_{4}$.

Proof. In the same way as in the proof of Lemma 2.1, we see that $U\left(\operatorname{cent}\left(Z S_{4} /(\bar{N})\right)\right)=\{ \pm 1\}$. Since the automorphism group of $S_{4}$ is isomorphic

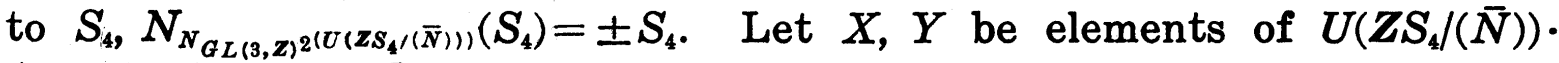
$\left(\left(E,\left[\begin{array}{rrr}1 & 2 & 2 \\ -2 & -3 & -2 \\ 2 & 2 & 1\end{array}\right]\right)\right)$. Then $X S_{4} X^{-1}$ and $Y S_{4} Y^{-1}$ are conjugate in $V_{o}\left(Z S_{4} /(\bar{N})\right)$ if and only if $Y^{-1} V_{o}\left(Z S_{4} /(\bar{N})\right) X \cap\left( \pm S_{4}\right) \neq \phi$. This condition is equivalent to $X^{-1} Y \in U_{0}\left(Z S_{4} /(\bar{N})\right)$. Therefore the number of conjugate classes in $V_{0}\left(Z S_{4} /(\bar{N})\right)$ of subgroups of $V_{0}\left(Z S_{4} /(\bar{N})\right)$ isomorphic to $S_{4}$ is $\left.\left[U\left(Z S_{4} /(\bar{N})\right) \cdot \mid\left(E,\left[\begin{array}{rrr}1 & 2 & 2 \\ -2 & -3 & -2 \\ 2 & 2 & 1\end{array}\right]\right)\right): U_{0}\left(Z S_{4} /(\bar{N})\right)\right]=8$.

THEOREM 3.5. There are 16 conjugate classes in $V\left(Z S_{4}\right)$ of subgroups of $V\left(Z S_{4}\right)$ isomorphic to $S_{4}$.

PRoof. Recall that $V\left(Z S_{4}\right)=\left\{\left(v_{1}, v_{2}\right) \in V_{0}\left(Z S_{4} /(\bar{N})\right) \times V\left(Z S_{3}\right) \mid g_{1}\left(v_{1}\right)=\right.$ $\left.g_{2}\left(v_{2}\right)\right\}$. Set $W=V_{o}\left(Z S_{4} /(\bar{N})\right) \times V\left(Z S_{3}\right)$. Then $N_{W}\left(S_{4}\right)=S_{4} \quad$ By Corollary 3.3, cent $\left(g_{1}\left(V\left(Z S_{3}\right)\right)\right)=1+2 \sigma+2 \sigma^{2}$, and hence $N_{W}\left(V\left(Z S_{4}\right)\right)=V\left(Z S_{4}\right)\langle 1,5-2 \sigma-$ $\left.\left.2 \sigma^{2}+4 \sigma \tau-4 \sigma^{2} \tau\right)\right\rangle$. Since there is 1 conjugate class in $V\left(Z S_{3}\right)$ of subgroups of $V\left(Z S_{3}\right)$ isomorphic to $S_{3}([3])$, the number of conjugate classes in $W$ of subgroups of $V\left(Z S_{4}\right)$ isomorphic to $S_{4}$ is 8 . Therefore the number of conjugate classes in $V\left(Z S_{4}\right)$ of subgroups of $V\left(Z S_{4}\right)$ isomorphic to $S_{4}$ is $8 \times\left[V\left(Z S_{4}\right) \cdot\left\langle\left(1,\left\langle 5-2 \sigma-2 \sigma^{2}+4 \sigma \tau-4 \sigma^{2} \tau\right\rangle\right)\right\rangle: V\left(Z S_{4}\right)\right]=16$.

\section{References}

[1] J. A. CoHN and D. Livingstone, On the structure of group algebras. I, Canad. J. Math.. 17 (1965), 583-593.

[2] R. K. DENNIs, The structure of the unit group of group rings, In: Ring Theory II, Marcel Dekker, New York 1977.

[3] I. Hughes and K. R. Pearson, The group of units of the integral group ring $Z S_{3}$, Canad. Math. Bull., 15 (1972), 529-534.

[4] D. A. JACKson, The groups of units of the integral group rings of finite metabelian and finite nilpotent groups, Quart. J. Math. Oxford, 2nd Series, 20 (1969), 319-331. 
[5] T. MrYata, On the units of the integral group ring of a dihedral group, to appear in J. Math. Soc. Japan.

[6] C. Polcino, The group of units of the integral group ring $Z D_{4}$, Bol. Soc. Brasil. Mat., 4 (1973), 85-92.

[7] I. ReINER and S. Ullom, A Mayer-Vietoris sequence for class groups, J. Algebra, 31 (1974), 305-342.

[8] K. TAHARA, On the finite subgroups of GL(3,Z), Nagoya Math. J., 41 (1971), 169-209.

[9] O. TAUSSKY, Matrices of rational integers, Bull. Amer. Math. Soc., 66 (1960), 327-345.

[10] S. UlLom, Nontrivial lower bounds for class groups of integral group rings, Illinois J. Math., 20 (1976), 361-371.

[11] A. Whiтсомв, The group ring problem, Ph. D. Thesis, University of Chicago, 1968.

Present Address:

Department of Mathematics

FACULTY OF ScIENCES

TOKYo Metropolitan University

Furazawa, Setagaya-ru, Tokyo 158 